\title{
Development of wideband circularly polarised square- and rectangular-loop antennas
}

\author{
R.L. Li, S. Basat, A. Traille, J. Laskar and M.M. Tentzeris
}

\begin{abstract}
New types of wideband circularly polarised (CP) square- and rectangular-loop antennas are developed and discussed. It is shown that the bandwidth for circular polarisation of a loop antenna can be significantly increased by adding a parasitic loop inside the original loop. The addition of the parasitic element can create one more minimum axial ratio (AR) point, which can appropriately combine with the original one, leading to a significant enhancement for the AR bandwidth. It is found that the AR $(\leq 2 \mathrm{~dB})$ bandwidth of a square-loop antenna can be increased from $6.5 \%$ to $20 \%$ by adding a parasitic square loop. By replacing the square loop with a rectangular loop, the AR bandwidth can be further enhanced. For a rectangular loop with an aspect ratio of $1 / 2$, a bandwidth of nearly $50 \%$ for $\mathrm{AR} \leq 2 \mathrm{~dB}$ is obtained with a gain of 8.5-7.5 dBi. A broadband balun is introduced for impedance matching.
\end{abstract}

\section{Introduction}

Square- and rectangular-loop antennas are usually used as linearly polarised antennas [1-5]. In recent years, it has been found that a square- or rectangular-loop antenna can also radiate a circularly polarised (CP) wave if a gap is introduced on the loop $[6,7]$. The reason for the $\mathrm{CP}$ radiation is due to the travelling-wave current distribution that becomes excited along the loop. An important feature of this type of $\mathrm{CP}$ antennas is that the sense of circular polarisation can be easily switched from left-hand to righthand, and vice versa, by altering the gap positions [8] using radio-frequency (RF) switches such as microelectromechanical systems (MEMS) [9], optoelectronic switches [10], or PIN diodes [11]. Although it is possible for a rectangularloop antenna to achieve a bandwidth close to $20 \%$ for an axial ratio (AR) of less than $3 \mathrm{~dB}$ [7], the bandwidths of square- and rectangular-loop antennas are still much less than those achieved by spiral antennas [12]. In this paper, it will be shown that the AR bandwidth of a CP square- or rectangular-loop antenna can be increased significantly by adding a parasitic loop (also with a gap) inside the original loop. The addition of the parasitic element can result in two minimum AR points that (by an appropriate combination) lead to a considerable enhancement for the AR bandwidth. As the additional parasitic element is placed inside the original loop and there is no direct electrical connection to its surroundings, there is no significant increase in the size and complexity of the antenna structure.

(C) The Institution of Engineering and Technology 2006

IEE Proceedings online no. 20050036

doi:10.1049/ip-map:20050036

Paper first received 18th February and in revised form 28th June 2005

The authors are with the Georgia Electronic Design Center, School of Electrical and Computer Engineering, Georgia Institute of Technology, Atlanta, GA 30332-0250, USA

E-mail: rlli@ece.gatech.edu

\section{Wideband square-loop antennas}

We begin the development of wideband CP square-loop antennas with a single square loop. Figure $1 a$ shows a square wire loop placed above a ground plane at a height $h$. The length of one side of the square loop is $a=0.3 \lambda_{0}$ and the wire radius is $r=0.008 \lambda_{0}$, where $\lambda_{0}$ is the free-space wavelength at a reference frequency $f_{0}$. The square loop is driven in the middle of one side by a voltage source $V_{0}$ (=1 volt). To produce CP waves, a small gap (the gap width $\left.\Delta s=0.02 \lambda_{0}\right)$ needs to be introduced on the loop [13]. The gap position and the height $h$ can be adjusted for an optimal on-axis (i.e. in the $z$ direction) AR performance. By numerical simulation, it was found that the optimised gap position should be at one of the two corners nearby the driving point. For example, a gap at the right corner corresponds to left-hand circular polarisation (LHCP). The sense of circular polarisation can be switched from the LHCP to right-hand circular polarisation (RHCP) by changing the gap position from the right corner to the left corner.

Figure 2 presents the simulated results of the on-axis AR as the height $h$ above the ground plane varies. (Note that almost all results shown in Sections 2 and 3 were obtained by numerical simulation using NEC 1.1, a method-ofmoment (MoM) based software.) The optimised height is found to be $h=0.225 \lambda_{0}$, which corresponds to a minimum AR close to $0 \mathrm{~dB}$ at $f_{0}$. It is noted that the bandwidth for $\mathrm{AR} \leq 2 \mathrm{~dB}$ of the single square-loop antenna is only about $6.5 \%$.

The AR bandwidth of a square loop can be enhanced by adding a parasitic element (a concentric square loop with a gap) inside the driven element (see Fig. 1b). Although a loop antenna with a parasitic element has been investigated before [14], the bandwidth for circular polarisation was not significantly improved. Here, the purpose for the addition of a parasitic square loop is to introduce a new minimum AR point. By adjusting the size of the parasitic loop and the position of its gap, it is possible to move the new minimum AR point (due to the parasitic loop) close to the original 


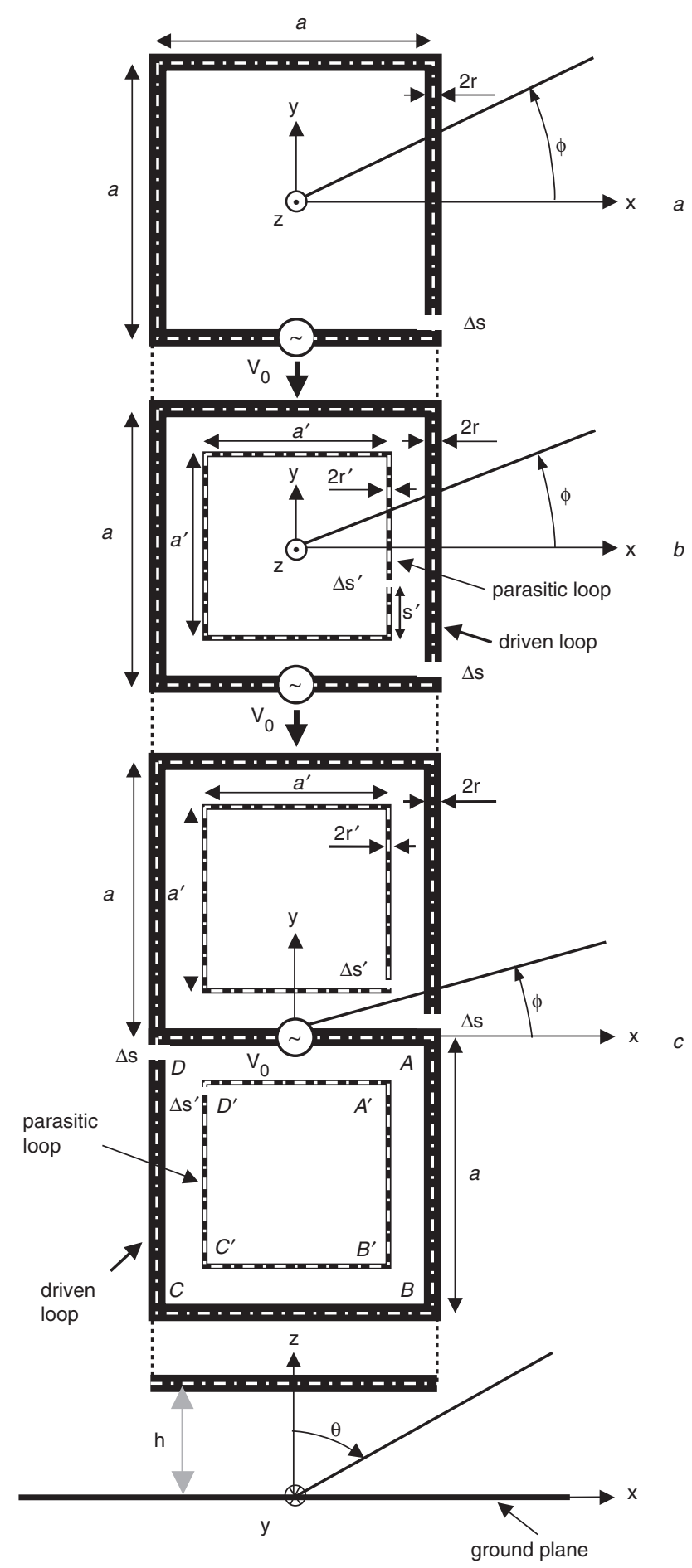

Fig. 1 Development of wideband circularly polarised square-loop antennas

$a$ Single loop

$b$ Single loop with a parasitic element

$c$ Twin loops with parasitic elements

minimum AR point (due to the driven loop). Note there is no need to change the size, the gap position and the height $h$ of the driven loop, despite the addition of the parasitic loop. A proper combination of the two minimum AR points leads to AR bandwidth enhancement. Figure 3 demonstrates how the side length $\left(a^{\prime}\right)$ and the gap position $\left(s^{\prime}\right)$ of the parasitic square loop affect the AR bandwidth performance. Indeed, two minimum AR points are observed at a lower (than the centre frequency $f_{\mathrm{c}}$ ) frequency $f_{\mathrm{L}}$ and at a higher frequency $f_{\mathrm{H}}$, respectively. As the

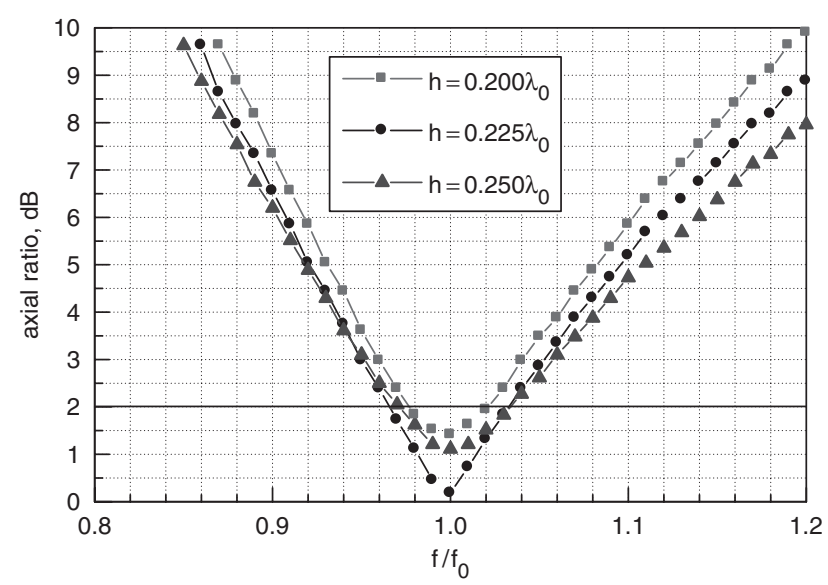

Fig. 2 Axial ratio of a square-loop antenna for different heights above a ground plane

Geometric parameters: $a=0.3 \lambda_{0}, r=0.008 \lambda_{0}$, and $\Delta s=0.02 \lambda_{0}$
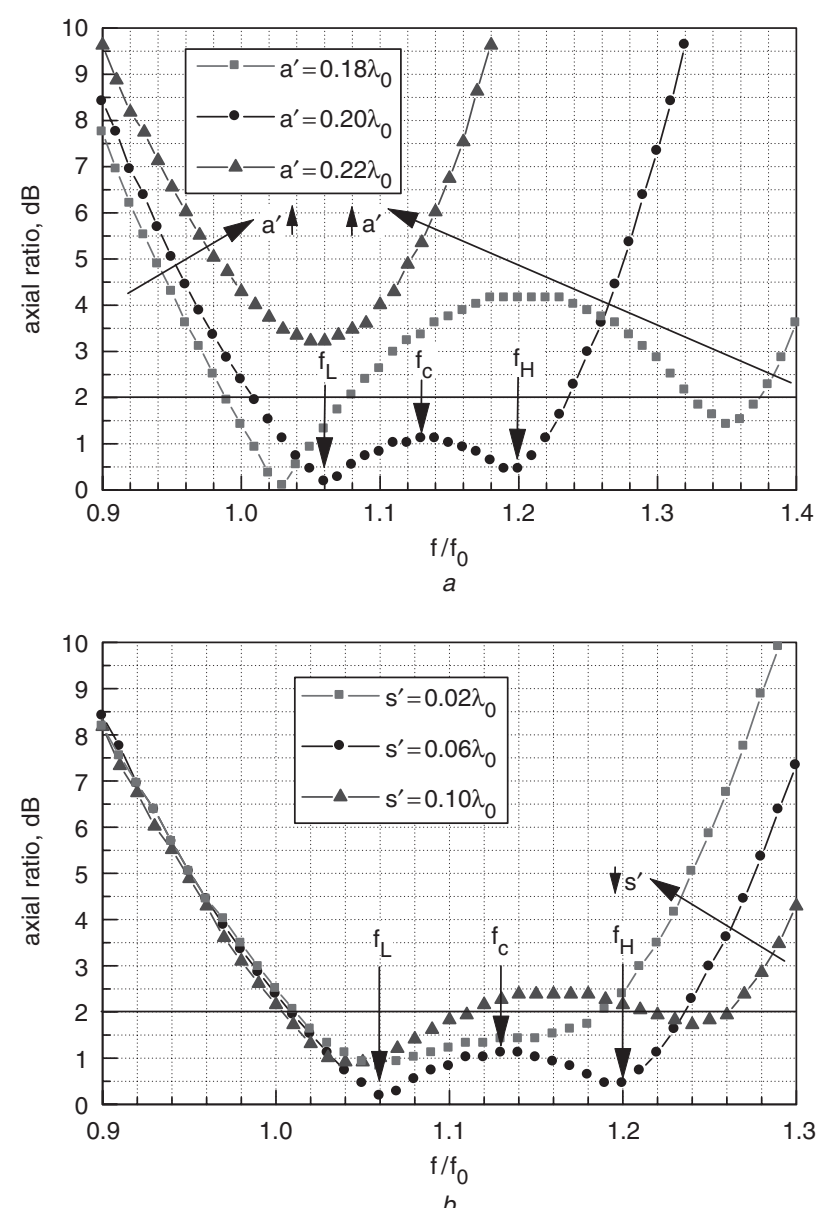

Fig. 3 Bandwidth enhancement for axial ratio of a square-loop antenna by adding a parasitic square loop

Geometric parameters: $a=0.3 \lambda_{0}, r=0.008 \lambda_{0}, r^{\prime}=0.004 \lambda_{0}, \Delta s=0.02 \lambda_{0}$, $\Delta s^{\prime}=0.01 \lambda_{0}$, and $h=0.225 \lambda_{0}$

$a$ With different side lengths of the parasitic loop $\left(s^{\prime}=0.06 \lambda_{0}\right)$

$b$ For different gap positions on the parasitic loop $\left(a^{\prime}=0.20 \lambda_{0}\right)$

side length increases $\left(a^{\prime} \uparrow\right)$ or the gap position moves toward the corner nearby the driving point $\left(s^{\prime} \downarrow\right)$, the two minimum AR points move closer to each other. If the side length is too long (e.g. $a^{\prime}>0.22 \lambda_{0}$ ) or the gap position is too close to the corner (e.g. $s^{\prime}<0.02 \lambda_{0}$ ), the two minimum AR 
points become overlapped and eventually retrograde to one minimum AR point. The optimised side length and gap position are found to be $a^{\prime}=0.20 \lambda_{0}$ and $s^{\prime}=0.06 \lambda_{0}$, respectively, which correspond to an optimal bandwidth of $20 \%$ for $A R \leq 2 \mathrm{~dB}$. It should be mentioned that it is difficult to enhance the AR bandwidth by placing the parasitic element outside the driven loop.

The bandwidth for circular polarisation can be further improved by introducing in cascade one more identical square loop to form a twin-square-loop antenna, as illustrated in Fig. 1c. One side of the introduced square loop is overlapped with a side of the original square loop and driven by the same voltage source. Also, a gap is introduced on the new square loop to achieve $\mathrm{CP}$ radiation and a parasitic element is added for bandwidth enhancement. The positions of the new gaps are opposite (with respect to the $z$ axis) to those of the original gaps, leading to a configuration similar to a figure-of-eight (or S-shaped) loop $[15,16]$, or a dual-spiral antenna $[17,18]$. It will be demonstrated that the twin-square-loop antenna also achieves a wider AR bandwidth. Figure 4 shows the dependence of AR performance of the twin-square-loop antenna on the gap position of the parasitic loops. The optimised gap position is at $s^{\prime}=0$, i.e. at the corner most nearby the driving point. Note that the rest of geometric parameters (such as $a, a^{\prime}$, and $h$ ) are kept unchanged after the introduction of one more square loop. The optimal AR bandwidth for $\mathrm{AR} \leq 2 \mathrm{~dB}$ is found to be $26 \%$. Figure 5 shows the frequency characteristics of the input impedance and gain of the twin-square-loop antenna with parasitic elements. The input resistance is about $150 \Omega$ and the gain is around $9.5 \mathrm{dBi}$.

The current distributions on the driven loop and on the parasitic loop of the twin-square-loop antenna are plotted in Fig. 6 at the centre frequency $f_{\mathrm{c}}=1.04 f_{0}$. A travelling-wave current distribution is observed on the driven loop. The current distribution on the parasitic loop manifests some properties between a travelling wave and a standing wave. The contribution to the $\mathrm{CP}$ radiation from the driven loop is dominant due its larger antenna size and higher current magnitude. There is no significant change for the current distribution at other frequencies in the $\mathrm{AR} \leq 2 \mathrm{~dB}$ bandwidth. Hence, a

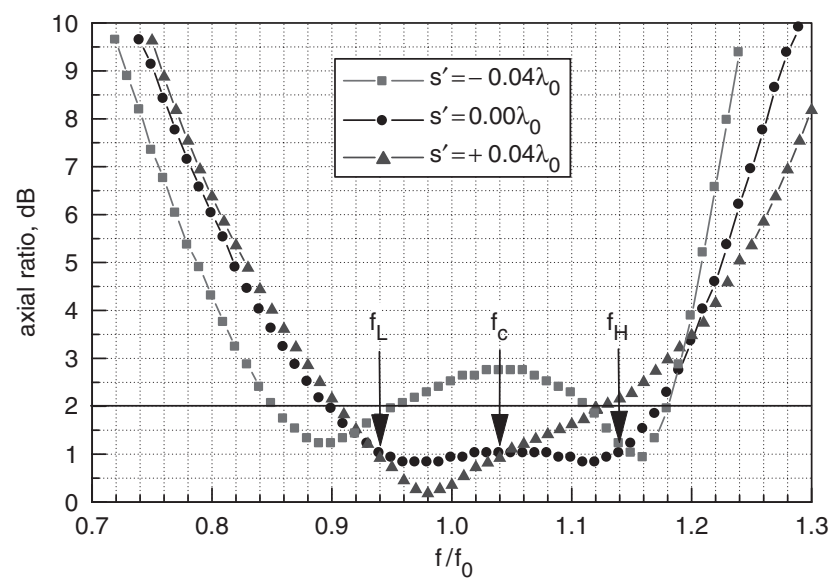

Fig. 4 Axial ratio of the twin-square-loop antenna for different gap positions on the parasitic loops

Geometric parameters: $a=0.3 \lambda_{0}, a^{\prime}=0.20 \lambda_{0}, r=0.008 \lambda_{0}, r^{\prime}=0.004 \lambda_{0}$, $\Delta s=0.02 \lambda_{0}, \Delta s^{\prime}=0.01 \lambda_{0}, h=0.225 \lambda_{0} ; s^{\prime}= \pm 0.04 \lambda_{0}$ means that the gap position moves $0.04 \lambda_{0}$ away ( + ) or toward (-) the driving point from the corner part of the role played by the parasitic element is probably to maintain a good travelling-wave current distribution on the driven loop over a wide range of frequencies, which, in

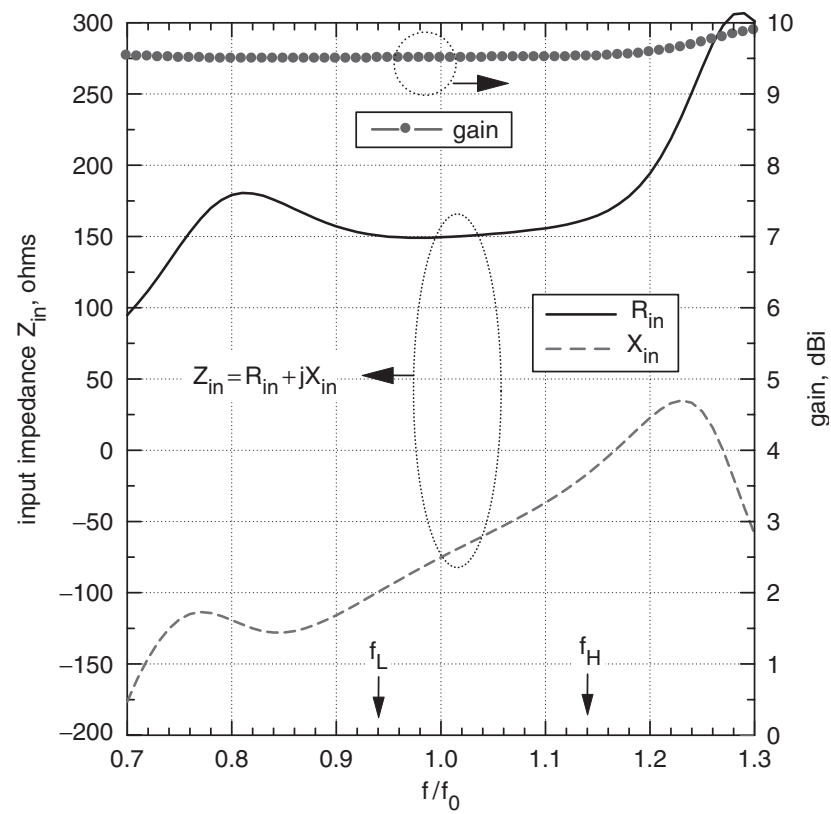

Fig. 5 Input impedance and gain of the twin-square-loop antenna with parasitic elements
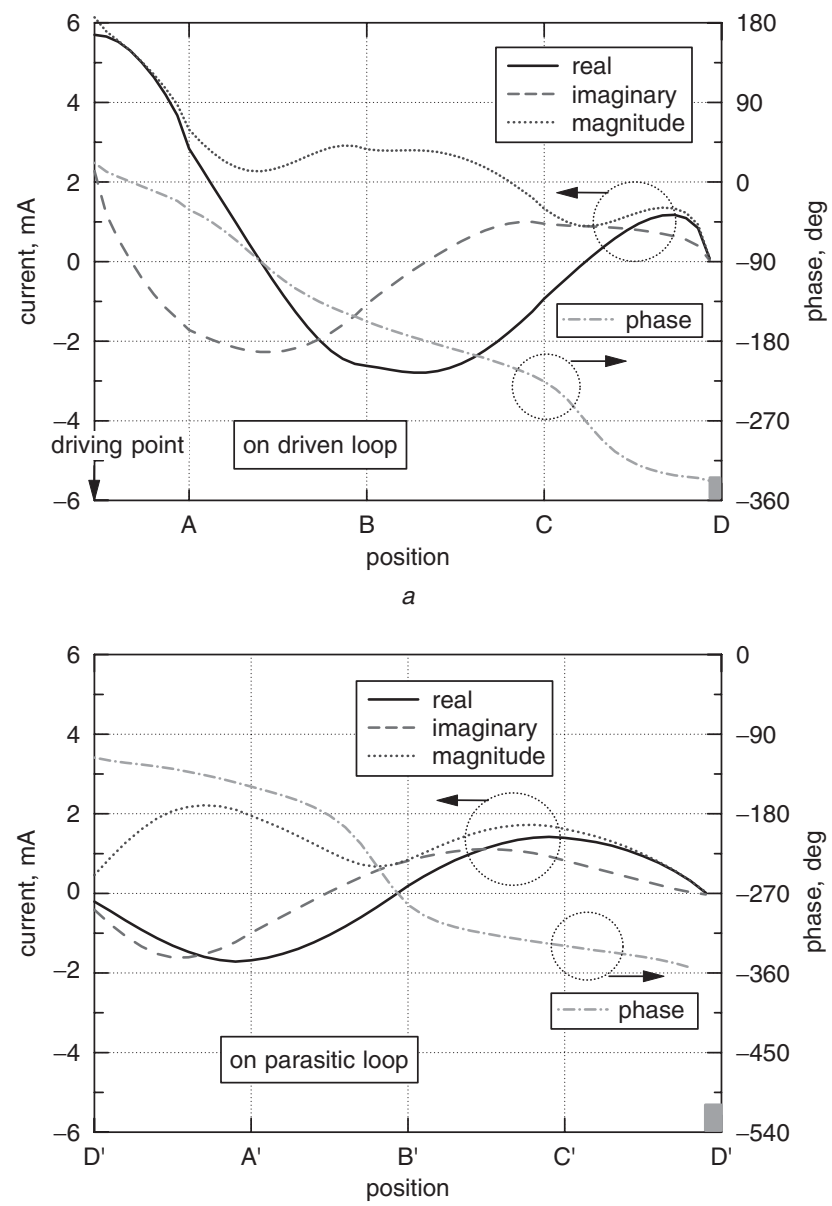

b

Fig. 6 Current distributions on the twin-square-loop antenna with parasitic elements $a$ On driven loop $b$ On parasitic loop 
turn, helps the loop antenna to achieve a wideband CP performance.

\section{Wideband rectangular-loop antennas}

The AR bandwidth of a square-loop antenna can be increased by replacing the square loop with a rectangular loop. In this Section, it will be demonstrated that the AR bandwidth can be increased to $50 \%$ by changing the aspect ratio (the width divided by the length) of a rectangular loop. Figure 7 illustrates the geometry of a twin-rectangular-loop antenna that consists of two driven rectangular loops, each with width $a$ and length $b$. A parasitic rectangular loop with width $a^{\prime}$ and length $b^{\prime}$ is introduced inside each of the driven loops. To keep the coupling between the driven loop and the parasitic loop as tight as possible, the distance between two adjacent sides of the loops is set to be a constant $d$, therefore the aspect ratio $a / b$ is not exactly equal to $a^{\prime} / b^{\prime}$ in general. The gap positions on both the driven loops and the parasitic loops need to be adjusted for an optimal AR performance. By simulation, it is found that as the aspect ratio of the rectangular loop decreases (i.e. the driven side becomes shorter), the gap positions need to move away from the corner near the driving point.

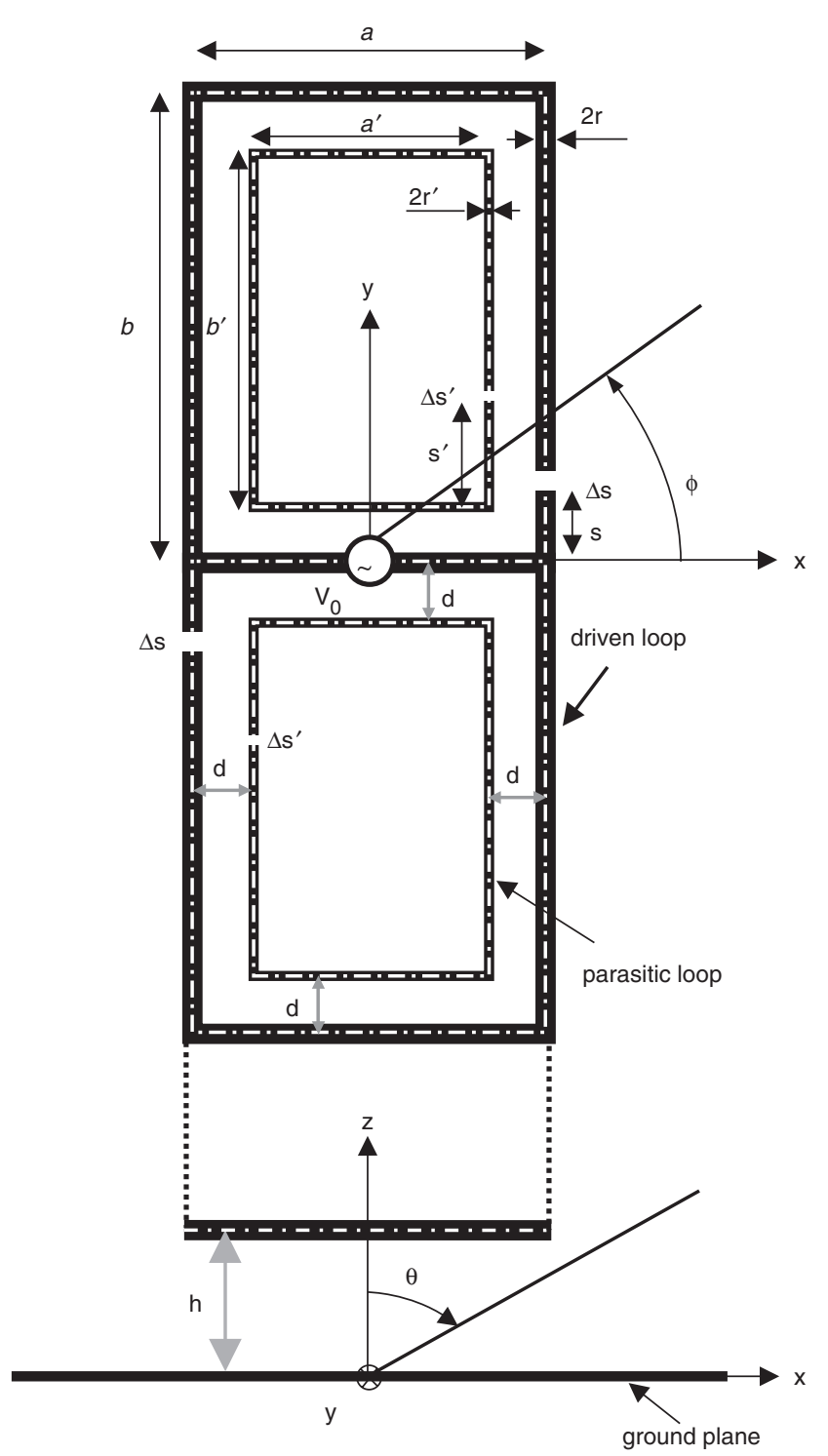

Fig. 7 Geometry of a wideband circularly polarised twinrectangular-loop antenna with parasitic elements
This is reasonable because the radiation field (i.e. $E_{x}$ in the $z$ direction) from the shorter sides of the rectangular loop decreases with the decrease in the aspect ratio. Correspondingly the radiation field (i.e. $E_{y}$ ) from the longer sides has to be reduced for good CP radiation. This can be achieved by moving the positions of the gaps away from the corner. Another parameter that needs to be adjusted is the height $h$ above the ground plane. It is found that, as the aspect ratio decreases, the height needs to be increased. The dependence of AR performance on the antenna height above a ground plane has also been observed for other planar $\mathrm{CP}$ antennas, such as spiral antennas backed by a conducting plane reflector [19]. Our investigation reveals that a decrease in the antenna height changes the travelling-wave current distribution on driven loops, thus deteriorating the AR performance.

To demonstrate the AR bandwidth enhancement by the replacement of rectangular loops, two different aspect ratios are investigated: $a / b=2 / 3$ and $a / b=1 / 2$. The perimeters of the driven rectangular loops and the parasitic rectangular loops keep the same as those of the square loops, i.e. $2(a+b)=1.2 \lambda_{0}$ and $2\left(a^{\prime}+b^{\prime}\right)=0.8 \lambda_{0}$. Figure 8 displays the AR variations as a function of frequency for the rectangular-loop antennas with aspect ratio $a / b=2 / 3$. The AR bandwidth of the single-rectangular-loop antenna without a parasitic element is only $8 \%(\mathrm{AR} \leq 2 \mathrm{~dB})$. By introducing the parasitic element, the AR bandwidth is increased to $23 \%$. The AR bandwidth for the twinrectangular-loop antenna with parasitic elements is found to be $36 \%$ (much wider than the $20 \%$ AR bandwidth achieved by the twin-square-loop antenna). This phenomenon is similar to that observed for a rhombic-loop antenna, where the AR bandwidth increases as the rhombus vertex angle decreases [13].

Figure 9 shows the AR bandwidth enhancement for the rectangular-loop antennas with aspect ratio $a$ / $b=1 / 2$. It is observed that the AR bandwidth for the singlerectangular-loop antenna without parasitic element is about $10 \%$. The introduction of a parasitic element increases the AR bandwidth to $29 \%$. For the twin-rectangular-loop antenna with parasitic elements, an $\mathrm{AR}(\leq 2 \mathrm{~dB})$ bandwidth of nearly $50 \%$ is achieved. To double check the achievement, we simulated the same antenna structure using Microstripes 6.5, a TLM (transmission-line matrix)

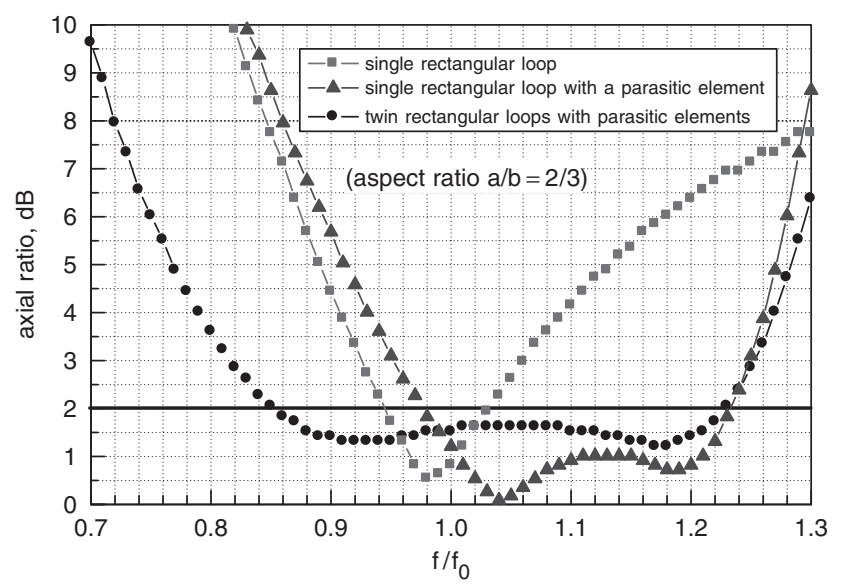

Fig. 8 Frequency characteristics of axial ratio for rectangular-loop antennas with aspect ratio alb $=2 / 3$

Geometric parameters: $a=0.24 \lambda_{0}, a^{\prime}=0.14 \lambda_{0}, b=0.36 \lambda_{0}, b^{\prime}=0.26 \lambda_{0}$, $r=0.008 \lambda_{0}, \quad r^{\prime}=0.004 \lambda_{0}, \quad \Delta s=0.02 \lambda_{0}, \quad \Delta s^{\prime}=0.01 \lambda_{0}, \quad s=0.04 \lambda_{0}$, $s^{\prime}=0.08 \lambda_{0}, d=0.05 \lambda_{0}$, and $h=0.25 \lambda_{0}$ 
based full-wave simulation tool. In the TLM-based simulation, the wire model used for the MoM-based simulation was replaced by a stripline with a width equal to four times the wire radius. Also a $0.665 \lambda_{0} \times 1.33 \lambda_{0}$ conducting copper plate was used as the ground plane in the TLM-based simulation. (Note that the size of ground plane was assumed to be infinite for the MoMbased simulation.) The TLM-based simulated AR is compared with the MoM-based simulated result in Fig. 9 and good agreement is observed. Note that the height of the twin-rectangular-loop antenna needs to be increased from $h=0.275 \lambda_{0}$ (of the single-rectangularloop antennas) to $h=0.30 \lambda_{0}$. It is also interesting to notice that the optimal distance $d$ between two adjacent sides of the loops is always around $0.05 \lambda_{0}$, no matter how much the aspect ratio is. By simulation, it is found that the $A R$

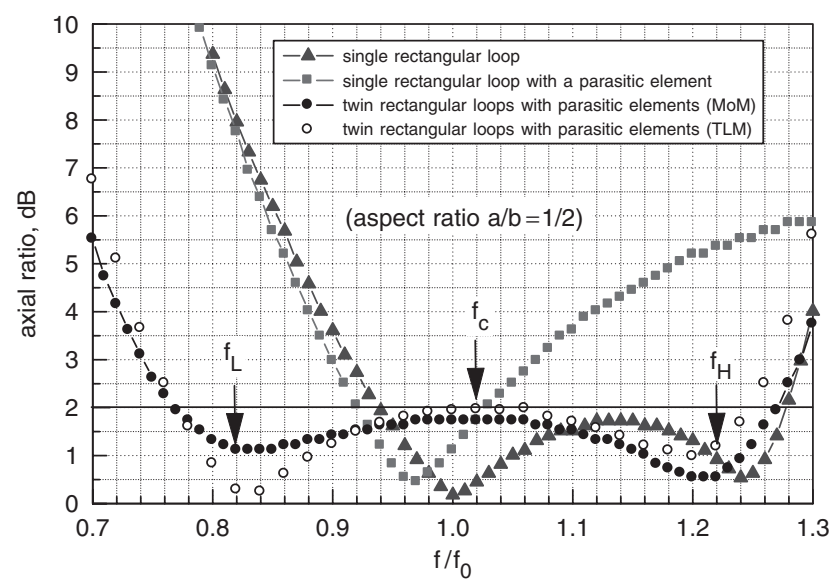

Fig. 9 Frequency characteristics of axial ratio for rectangular-loop antennas with aspect ratio alb $=1 / 2$

Geometric parameters: $a=0.2 \lambda_{0}, a^{\prime}=0.1 \lambda_{0}, \quad b=0.4 \lambda_{0}, \quad b^{\prime}=0.3 \lambda_{0}$, $r=0.008 \lambda_{0}, \quad r^{\prime}=0.004 \lambda_{0}, \quad \Delta s=0.02 \lambda_{0}, \quad \Delta s^{\prime}=0.01 \lambda_{0}, \quad s=0.06 \lambda_{0}$, $s^{\prime}=0.12 \lambda_{0}, \quad d=0.05 \lambda_{0}, h=0.275 \lambda_{0}$ for the single-rectangular-loop antennas, and $h=0.3 \lambda_{0}$ for the twin-rectangular-loop antenna

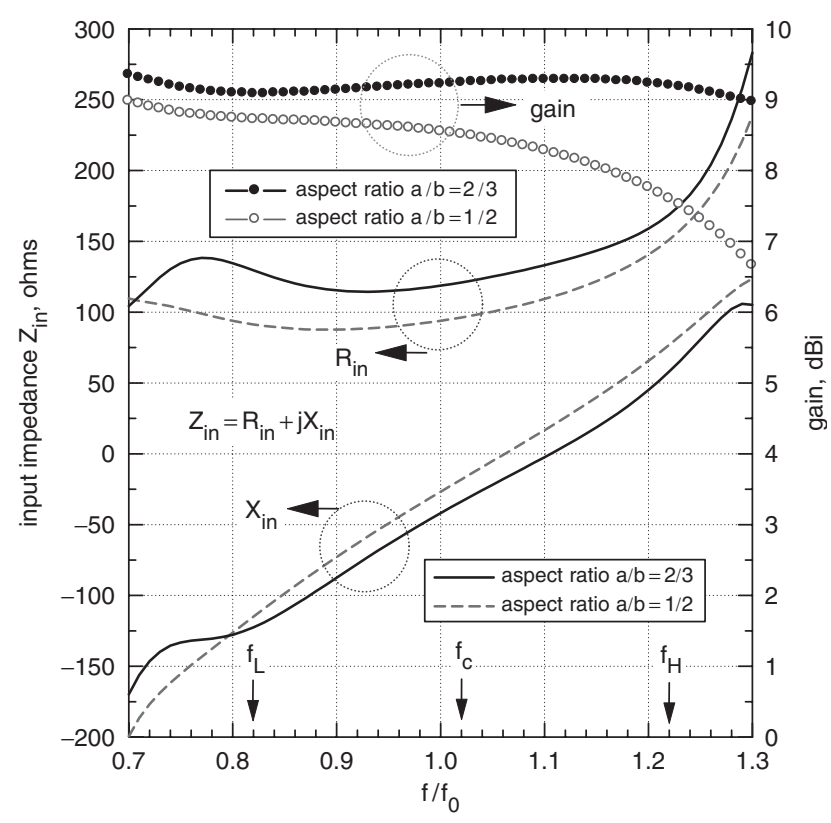

Fig. 10 Input impedance and gain of the twin-rectangular-loop antennas with parasitic elements performance of the loop antennas is not very sensitive to the wire radii (i.e. $r$ and $\left.r^{\prime}\right)$ of the loops and the gap widths $(\Delta s$ and $\Delta s^{\prime}$ ). As a rule of thumb, it is suggested that we choose $r=0.01-0.005 \lambda_{0}, r^{\prime}=0.4-0.6 r$ and the gap widths equal to 2-4 times the wire radii [8]. As the gap widths are much smaller than the loop size, it is possible to replace the gaps with RF switches, such as MEMS or PIN diodes to achieve polarisation configuration.

The frequency characteristics of the input impedance and gain of the twin-rectangular-loop antennas are displayed in Fig. 10. The input resistance decreases as the aspect ratio decreases. This tendency is similar to the variation of resonant resistance for a linearly polarised rectangular-loop antenna [20]. The gain slightly decreases as the aspect ratio decreases $(\sim 9 \mathrm{dBi}$ for $a / b=2 / 3$ against $8.5-7.5 \mathrm{dBi}$ for $a / b=1 / 2)$ due to the increased height $h$ above the ground plane $\left(h=0.25 \lambda_{0}\right.$ for $a / b=2 / 3$ against $h=0.3 \lambda_{0}$ for $a / b=$ $1 / 2$ ) [21]. If the aspect ratio further decreases, the $A R$ $(\leq 2 \mathrm{~dB}$ ) bandwidth may be more than $50 \%$, but the gain will decrease considerably as the height needs to be further increased. As an example, we examined the case for aspect ratio $a / b=1 / 3$. The AR $(\leq 2 \mathrm{~dB})$ bandwidth was found to be approximately $60 \%$ with an optimised antenna height $h=0.38 \lambda_{0}$, but the on-axis gain became $\sim 6 \mathrm{dBi}$ at the centre frequency $f_{\mathrm{c}} \cong 1.0 f_{0}$ and $\sim-10 \mathrm{dBi}$ at the higher frequency $f_{\mathrm{H}} \cong 1.3 f_{0}$, because the maximum radiation no longer occurs in the $z$ direction.

\section{An experimental example}

To demonstrate the bandwidth enhancement experimentally, a twin-rectangular-loop antenna with an aspect ratio close to $2 / 3$ was fabricated and measured. The reason for selecting this aspect ratio is that the bandwidth of our antenna-radiation-pattern measurement system is less than $36 \%$. The antenna measurement system employed is the NSI near-field system which is capable of measuring amplitude and phase patterns. The used probe (as a TX source antenna) is a WR159 open-ended waveguide probe that covers the frequency range $4.9-7.05 \mathrm{GHz}$, therefore we chose $6 \mathrm{GHz}$ as the centre frequency. The configuration and dimensions of the printed twin-rectangular-loop antenna are illustrated in Fig. 11. The twin rectangular loops with parasitic elements are printed on a thin dielectric substrate (substrate thickness $=0.254 \mathrm{~mm}$ ) with a low dielectric constant (RT/duroid 5880, $\left.\varepsilon_{\mathrm{r}}=2.2\right)$. The printed twin-rectangular-loop antenna is mounted $13 \mathrm{~mm}$ above a $40 \mathrm{~mm} \times 60 \mathrm{~mm}$ copper plate. The antenna is fed by a broadband balun which was fabricated on the same type of RT/duroid substrate as used for the printed loop (namely, $\varepsilon_{\mathrm{r}}=2.2$ and thickness $=0.254 \mathrm{~mm}$ ). This type of broadband balun has been widely used for broadband printed dipole antennas [22-24] and, to our knowledge, however, this is the first time for it to be used for a CP loop antenna. The broadband balun can excite the balanced mode by making use of the coupling between the microstripline printed on one side of the substrate to the slot which is etched on the other side (which also serves as the ground plane for the microstripline) of the substrate. A good impedance matching can be achieved by adjusting the length $l_{\mathrm{s}}$ of the slot, the height $h_{\mathrm{m}}$ and the length $l_{\mathrm{m}}$ of the microstripline. Figure 12 presents the simulated and measured results for voltage standing-wave ratio (VSWR) of the broadband balun fed twin-rectangular-loop antenna, which shows a broadband impedance bandwidth of $\sim 50 \%$ $(\mathrm{VSWR} \leq 2)$. 


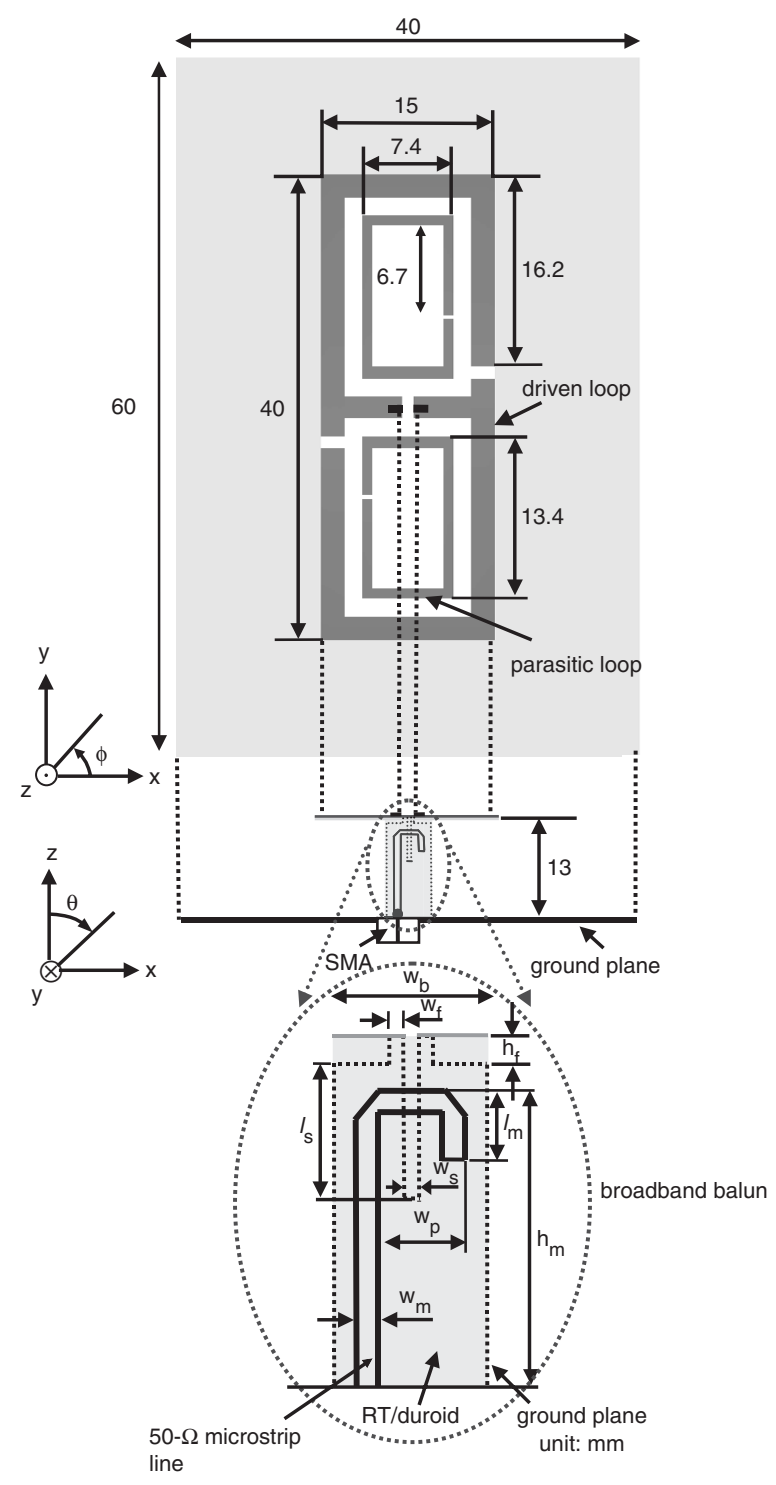

Fig. 11 Printed twin-rectangular-loop antenna with parasitic elements

The strip widths of the driven loops and of the parasitic loops are $2.0 \mathrm{~mm}$ and $1.0 \mathrm{~mm}$, respectively; the gap widths on the driven loops and on the parasitic loops are $1.0 \mathrm{~mm}$ and $0.5 \mathrm{~mm}$, respectively; $w_{\mathrm{b}}=6.0 \mathrm{~mm}, w_{\mathrm{f}}=0.6 \mathrm{~mm}, w_{\mathrm{s}}=0.5 \mathrm{~mm}, w_{\mathrm{m}}=0.8 \mathrm{~mm}, w_{\mathrm{p}}=3.2 \mathrm{~mm}$, $h_{\mathrm{f}}=1.0 \mathrm{~mm}, h_{\mathrm{m}}=11 \mathrm{~mm}, l_{\mathrm{s}}=5 \mathrm{~mm}, l_{\mathrm{m}}=2.5 \mathrm{~mm}$

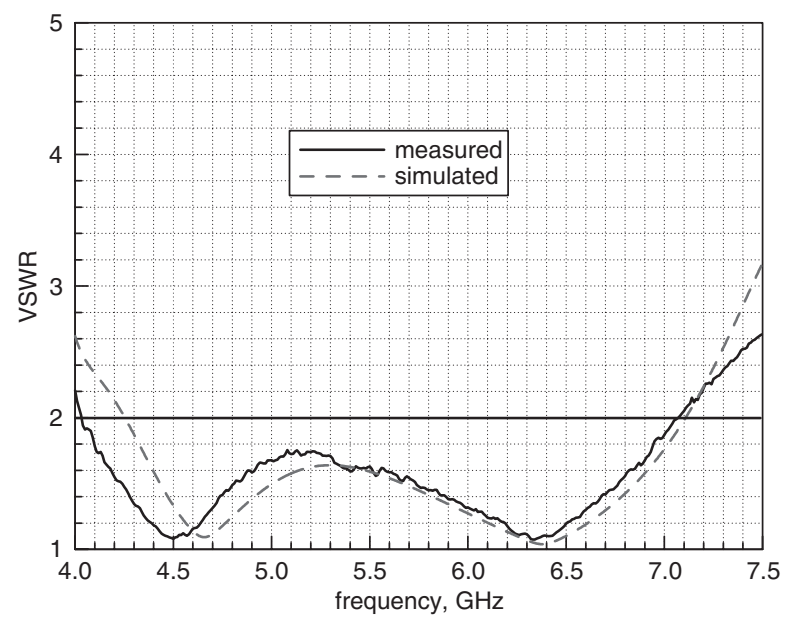

Fig. 12 VSWR of printed twin-rectangular-loop antenna with parasitic elements

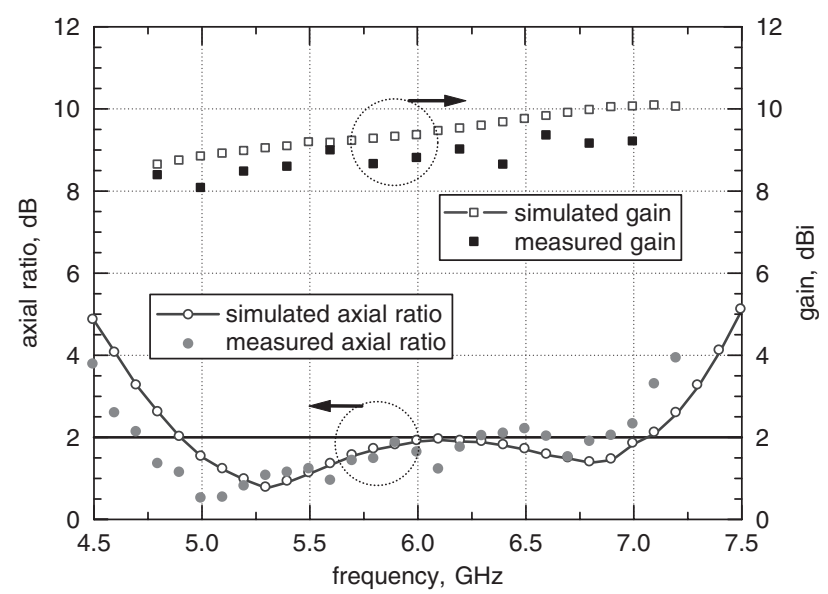

Fig. 13 Axial ratio and gain of printed twin-rectangular-loop antenna with parasitic elements

Figure 13 presents the simulated and measured results for the axial ratio and gain, showing good agreement. The AR bandwidth for AR $\leq 2 \mathrm{~dB}$ is found to be about $34 \%$. The measured gain is around $9 \mathrm{dBi}$ over the $\mathrm{AR} \leq 2 \mathrm{~dB}$ bandwidth. The radiation patterns simulated and measured at $5.0 \mathrm{GHz}, 6.0 \mathrm{GHz}$ and $7.0 \mathrm{GHz}$ are compared in Fig. 14. Good agreement is obtained in the main beam of the copolarisation (i.e. LHCP). As expected, the beamwidth $\left(\sim 75^{\circ}\right)$ in $\phi=0^{\circ}$ cut (the $x-z$ plane) is wider than that $\left(\sim 50^{\circ}\right)$ in $\phi=90^{\circ}$ cut (the $y-z$ plane), because the antenna structure is two times longer in the $y$ direction than that in the $x$ direction. The backlobe level and the cross-polarised radiation (i.e. $\mathrm{RHCP}$ ) are less than $-15 \mathrm{~dB}$.

\section{Conclusion}

A new type of wideband $\mathrm{CP}$ square- and rectangular-loop antennas has been developed and extensively discussed. It has been demonstrated that the bandwidth for circular polarisation of a loop antenna can be significantly increased by incorporating a parasitic loop inside the original loop. The incorporated loop serves as a parasitic element and introduces an additional minimum AR point. A proper combination of the original and additional minimum AR points leads to an AR bandwidth enhancement. It has been found that the AR $(\leq 2 \mathrm{~dB})$ bandwidth of a square-loop antenna with a parasitic square loop can be increased from $6.5 \%$ to $20 \%$. The AR bandwidth of a single-loop antenna can be improved by combining two loops into a twin-loop antenna. The gain and AR bandwidth of a twin-square-loop antenna is found to be $9.5 \mathrm{dBi}$ and $26 \%$ for $\mathrm{AR} \leq 2 \mathrm{~dB}$, respectively. The $\mathrm{AR}$ bandwidth of the twin-square-loop antenna is further enhanced by replacing the square loop with a rectangular loop. A bandwidth of approximately $50 \%$ for AR $\leq 2 \mathrm{~dB}$ is obtained with a gain of $8.5-7.5 \mathrm{dBi}$ for the twinrectangular-loop antenna with an aspect ratio of $1 / 2$. The impedance matching has been achieved by introducing a broadband balun. As the sense of circular polarisation of the wideband CP loop antennas is controlled by the positions of several small gaps on the loops, it is possible to realise polarisation configuration (e.g. to change LHCP to RHCP, and vice versa) using RF switches such as MEMS or PIN diodes. 

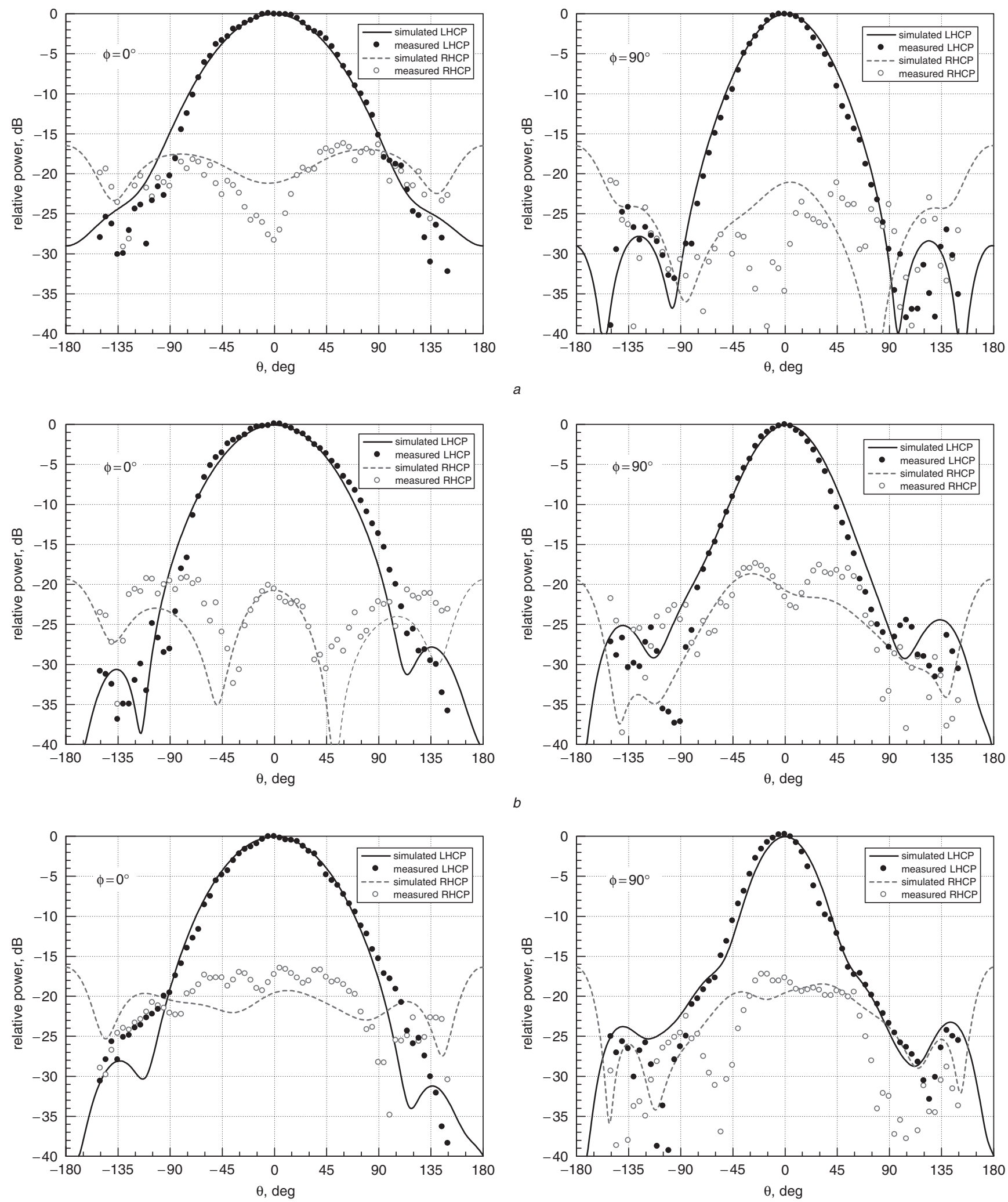

Fig. 14 Radiation patterns of printed twin-rectangular-loop antenna with parasitic elements a $f=5.0 \mathrm{GHz}$

$b f=6.0 \mathrm{GHz}$

$c f=7.0 \mathrm{GHz}$

\section{Acknowledgments}

The authors wish to acknowledge the support of the Georgia Electronic Design Centre (GEDC), the NSF CAREER Award ECS-9984761, the NSF Grant ECS0313951 and the NSF Packaging Research Centre.

\section{References}

1 Stutzman, W.L., and Thiele, G.A.: 'Antenna theory and design' ( John Wiley \& Sons, Inc., 1998), pp. 205-210

2 King, R.: 'The rectangular loop antenna as a dipole', IRE Trans. Antennas Propag., 1959, 7, (1), pp. 53-61

3 Tsukiji, T.: 'Analysis of two coupled coplanar loops', IEEE Trans. Antennas Propag., 1975, 23, (2), pp. 250-253 
4 Jensen, M.A., and Rahmat-Samii, Y.: 'Electromagnetic characteristics of superquadric wire loop antennas', IEEE Trans. Antennas Propag., 1994, 42, (2), pp. 264-269

5 Levia, B.: 'Field of a rectangular loop', IEEE Trans. Antennas Propag., 2004, 52, (4), pp. 948-952

6 Shi, S., Hirasawa, K., and Chen, Z.N.: 'Circularly polarized rectangularly bent slot antennas backed by a rectangular cavity', IEEE Trans. Antennas Propag., 2001, 49, (11), pp. 1517-1524

7 Sumi, M., Hirasawa, K., and Shi, S.: 'Two rectangular loops fed in series for broadband circular polarization and impedance matching', IEEE Trans. Antennas Propag., 2004, 52, (2), pp. 551-554

8 Morishita, H., Hirasawa, K., and Nagao, T.: 'Circularly polarised wire antenna with a dual rhombic loop', IEE Proc., Microw. Antennas Propag., 1998, 145, (3), pp. 219-224

9 Weedon, W.H., Payne, W.J., and Rebeiz, G.M.: 'MEMS-switched reconfigurable antennas'. Proc. IEEE AP-S Int. Symp., 2001, Vol. 3, pp. 654-657

10 Daryoush, A.S., and Choe, B.: 'Optically reconfigured active phased array antennas'. Proc. IEEE AP-S Int. Symp., 1988, Vol. 1, pp. $44-47$

11 Chang, B.C.C., Qian, Y., and Itoh, T.: 'A reconfigurable leaky mode/ patch antenna controlled by PIN diode switches'. Proc. IEEE AP-S Int. Symp., 1999, Vol. 4, pp. 2694-2697

12 Afsar, M.N., Wang, Y., and Cheung, R.: 'Analysis and measurement of a broadband spiral antenna', IEEE Antennas Propag. Mag., 2004, 46, (1), pp. 59-64

13 Morishita, H., and Hirasawa, K.: 'Wideband circularly-polarised loop antenna'. Proc. IEEE AP-S Int. Symp., 1994, pp. 1286-1289
14 Nakano, H., Mimaki, H., Wu, Y., Nakayama, K., and Yamauchi, J.: 'Loop antenna with a parasitic element'. Proc. Symp. Antenna Technol. Appl. Electromagn. (ANTEM), 1994, pp. 419-422

15 Li, R.L., and Fusco, V.: 'Printed figure-of-eight wire antenna for circular polarization', IEEE Trans. Antennas Propag., 2002, 50, (10), pp. $1487-1490$

16 Morishita, H., Hamada, H., Nishida, K., and Nagoa, T. "A wideband circularly polarized dipole antenna'. Proc. IEEE AP-S Int. Symp., 1998, pp. 2348-2350

17 Hirose, K., and Nakano, H.: 'Dual-spiral slot antennas', IEE Proc. H, Microw. Antennas Propag., 1991, 138, (1), pp. 32-36

18 Nakano, H., and Hirose, K. 'Numerical analysis of a dual-spiral printed antenna'. Proc. IEE 8th Int. Conf. Antennas Propag., ICAP, 1993, pp. 651-654

19 Nakano, H., Nogami, K., Arai, S., Mimaki, H., and Yamauchi, J.: 'A spiral antenna backed by a conducting plane reflector', IEEE Trans. Antennas Propag., 1986, 34, (6), pp. 791-796

20 Li, R.L., Ni, G., and Nakano, H.: 'Numerical analysis of printed superquadric wire loop antennas', IEEE Trans. Magn., 1998, 34, (5), pp. 2787-2790

21 Balanis, C.A.: 'Antenna theory: analysis and design' (John Wiley \& Sons, Inc., 1997), pp. 175-181

22 Edward, B., and Rees, D.: 'A broadband printed dipole with integrated balun', Microwave J., 1987, pp. 339-344

23 Leong, K.M.K.H., Qing, Y., and Itoh, T.: 'Surface wave enhanced broadband planar antenna for wireless applications', IEEE Microw. Wirel. Components Lett., 2001, 11, (2), pp. 62-64

24 Sabatier, C.: 'T-dipole arrays for mobile applications', IEEE Antennas Propag. Mag., 2003, 45, (6), pp. 9-26 